\title{
En prevención primaria, los riesgos de la TRH combinada exceden sus beneficios
}

Risks and benefits of estrogen plus progestin in healthy postmenopausal women. Results of the Women's Health Initiative randomized controlled trial. JAMA 2002, 288:321-333

\section{Objetivo}

Evaluar los beneficios y riesgos principales de la terapia de reemplazo hormonal (TRH) combinada.

Diseño

El componente Estrógeno más Progesterona de la iniciativa de Salud de la Mujer de EE.UU. (Women Health Initiative).Ensayo clínico controlado aleatorizado de prevención primaria con un seguimiento planeado de 8.5 años, en el que se incluyeron 16.608 mujeres post-menopáusicas de entre 50-79 años con un útero intacto al inicio del estudio.

Lugar

Cuarenta centros en EE.UU.

Intervención

Estrógenos equinos conjugados (EEC), $0.625 \mathrm{mg} /$ día más acetato de medroxiprogesterona (AMP) $2.5 \mathrm{mg} /$ día, en una tableta $(n=8506)$ o placebo $(n=8102)$.

\section{Medición de resultados principales}

El resultado primario fue enfermedad coronaria (EC) (infarto de miocardio no fatal y muerte por EC), con cáncer de mama invasivo como el resultado adverso primario.Se construyó un índice global sumario que incluía beneficios y riesgos (los dos resultados primarios más accidente cerebrovascular [ACV], tromboembolismo pulmonar [TEP], cáncer de endometrio, cáncer colorrectal, fractura de cadera, y muerte por otras causas).

\section{Resultados}

El estudio fue suspendido tempranamente a los 5.2 años de seguimiento debido a una recomendación del comité de vigilancia ya que se observó un exceso de cáncer de mama invasivo y el índice global apoyaba que los riesgos excedían los beneficios. No hubo diferencias en la mortalidad global ni por causas específicas; tampoco en los cánceres totales.
Tabla.Principales resultados

\begin{tabular}{l|c|c|c|c}
\hline Resultado & \multicolumn{2}{|c|}{$\begin{array}{c}\text { Nro de pacientes } \\
\text { (anualizado \%) }\end{array}$} & $\begin{array}{c}\text { Riesgo Relativo } \\
\text { (IC 95\%) }\end{array}$ & $\begin{array}{c}\text { NND 0 } \\
\text { NNT }\end{array}$ \\
\hline & $\begin{array}{c}\mathrm{E}+\mathrm{P} \\
(8506)\end{array}$ & $\begin{array}{c}\text { Placebo } \\
(8506)\end{array}$ & & \\
\hline EC & $164(0.37)$ & $122(0.30)$ & $1.29(1.02-1.63)$ & 1428 \\
\hline ACV & $127(0.29)$ & $85(0.21)$ & $1.41(1.07-1.85)$ & 1250 \\
\hline Eventos Tromboembólicos@ & $151(0.34)$ & $67(0.16)$ & $2.11(1.58-2.82)$ & 556 \\
\hline Cáncer de mama invasivo & $166(0.38)$ & $124(0.30)$ & $1.26(1.00-1.59)$ & 1250 \\
\hline Cáncer colorrectal & $45(0.10)$ & $67(0.16)$ & $0.63(0.43-0.92)$ & 1667 \\
\hline Fracturas de cadera & $44(0.10)$ & $62(0.15)$ & $0.66(0.45-0.98)$ & 2000 \\
\hline Muertes & $231(0.52)$ & $218(0.53)$ & $0.98(0.82-1.18)$ & - \\
\hline Indice Global@@ & $751(1.70)$ & $623(1.51)$ & $1.15(1.03-1.28)$ & 526 \\
\hline
\end{tabular}

@ incluyen al TEP y a la trombosis venosa profunda, @@ el primero de cualquiera de los siguientes eventos:EC, cáncer de mama invasivo, ACV, TEP, cáncer de endometrio, cáncer colorrectal, fractura de cadera, y muerte por otras causas.

El incremento de riesgo absoluto por cada 10.000 pacientes por año atribuible a la TRH combinada es de 7 eventos coronarios más, 8 ACV más, 8 TEP más, y 8 cánceres invasivos de mama más.La reducción de riesgo absoluto por cada 10.000 pacientes por año fueron 6 cánceres colorrectales menos y 5 fracturas de cadera menos. El exceso absoluto de eventos globales fue de 19 cada 10.000 personas/año.

\section{Conclusiones}

En un promedio de 5.2 años de seguimiento, los riesgos para la salud de la TRH combinada superaron los beneficios en mujeres postmenopáusicas sanas.La mortalidad global fue similar.

Fuente de financiamiento:no referida.

\section{Comentario}

Durante la última década, el uso de terapia de reemplazo hormonal (TRH) en mujeres post menopáusicas se incorporó progresivamente a la práctica médica. En algunos lugares del mundo, como en los EE.UU, algunas estadísticas reportaron que casi la mitad de las mujeres post menopáusicas recibían o habían recibido TRH.

Aún cuando las indicaciones aprobadas por la FDA, (Food and Drug Administration) son el manejo de los síntomas postmenopáusicos y la prevención de osteoporosis, la utilización a largo plazo principalmente para prevención de enfermedades crónicas se fue imponiendo a medida que los estudios disponibles sugerían beneficios sustanciales en la reducción de la enfermedad coronaria y/o la muerte de origen cardiovascular, aunque también sugerían un aumento en el riesgo de cáncer de mama1,2.

Muchos investigadores criticaron estos resultados ya que provenían de estudios de cohortes, los que, aunque estuvieran bien diseñados y conducidos, no escapaban a la influencia de posibles sesgos. La publicación del HERS en 199833 el primer ensayo clínico aleatorizado de TRH combinada en prevención secundaria de eventos cardiovasculares, fue la primera luz de alarma, al demostrar que el beneficio sugerido por los estudios observacionales en la población de mayor riesgo, (aquellas mujeres con enfermedad coronaria establecida), no se confirmaba.La cantidad de eventos sufridos fue la misma para el grupo TRH y el grupo placebo. Al mismo tiempo se observó un aumento del riesgo de los fenómenos tromboembólicos venosos, (TVP y TEP), de 3 a 1, aún cuando la diferencia absoluta de riesgo fue pequeña, (de $0,7 \%$ a casi $2 \%$ en 4 años).

Al final del primer año se registró un número mayor de eventos cardiovasculares en el grupo TRH respecto al grupo placebo, (RR 1er año $=1.52$, IC95\% 1.01-2.29), mientras que a lo largo del estudio y al final de los años cuarto y quinto los eventos fueron más numerosos en el grupo placebo, $(R R=0.67, I C 95 \%$ 0.46-0.98). Se ensayaron varias explicaciones para estos hallazgos basándose en el efecto protrombótico y proisquémico atribuido a las hormonas, en especial a la medroxiprogesterona.Como la tendencia temporal podía llegar a mostrar un posible beneficio a más largo plazo, se decidió la continuación de este estudio por casi 3 años más en el llamado HERS II y resumido en estas páginas.

Este estudio da por tierra con la indicación de TRH combinada a largo plazo para la prevención secundaria de enfermedad coronaria, ya que el seguimiento global a casi 7 años no mostró diferencias en cuanto al número de eventos en uno y otro grupo.

Dos semanas más tarde, se publican los primeros resultados del esperado ensayo de la TRH combinada en prevención primaria, el Women's Health Initiative. Los métodos reportados son impecables y sus resultados, al menos, desalentadores.

EI WHI se concentró en analizar el riesgo cardiovascular y el de cáncer de mama y tuvo que ser detenido precozmente cuando en el décimo análisis interino el riesgo de cáncer de mama superó la diferencia preestablecida en el diseño como valor umbral para suspender el estudio.

Dado que la población estaba constituida por mujeres de bajo riesgo, (prevención primaria), el número de eventos ocurridos (cardiovasculares, cánceres, fracturas) durante los 5 años fue en general bajo.

En cuanto al aumento de eventos coronarios, estos se concentraron en los infartos de miocardio no fatales (la mortalidad no fue distinta), y se empieza a observar desde el comienzo del estudio. El aumento del cáncer de mama se observó a partir de los 3,5 años de uso, lo cual es consistente con los hallazgos de otros muchos estudios.

Tanto los eventos tromboembólicos como los accidentes cerebrovasculares (ACV), fueron más frecuentes en el grupo TRH.Mientras que en los primeros el efecto se observó desde el comienzo del estudio, para los segundos recién se empezó a notar luego del año y medio.

Los beneficios definitivos de la TRH se apreciaron en la menor incidencia de fracturas (observada desde el comienzo) y de cáncer de colon en el grupo tratado (luego de tres años de seguimiento).

Es de hacer notar que los presentes estudios no reportan el control de los síntomas vasomotores y genitourinarios, ya confirmados en otros ensayos clínicos. 


\section{La TRH combinada no fue beneficiosa a largo plazo en prevención secundaria}

Cardiovascular disease outcome during 6.8 years of hormone therapy. Heart and Estrogen / Progestin replacement Study (HERS II). Grady D, Herrington D, Bittner V et al. JAMA 2002, 288:49-57

\section{Objetivo}

Determinar si la reducción de riesgo observada en los últimos años del estudio HERS persistió y resultó en una reducción global del riesgo de eventos de enfermedad coronaria (EC) en los años subsiguientes. Diseño

Ensayo clínico controlado aleatorizado doble ciego, de 4.1 años de duración (HERS), y seguimiento subsecuente por 2.7 años más en forma abierta.

Lugar

Veinte centros ambulatorios y comunitarios de EE.UU.

Pacientes

Se incluyeron 2.763 mujeres posmenopáusicas con EC y una edad promedio de 67 años al inicio del HERS.El 93\% de las sobrevivientes consintieron ingresar al HERS II $(n=2321)$.

Intervención

HERS: 1380 pacientes recibieron estrógenos equinos conjugados (EEC) $0.625 \mathrm{mg} /$ día más $2.5 \mathrm{mg} /$ día de acetato de medroxiprogesterona (AMP) y 1383 recibieron placebo.HERS II:Se prescribió abiertamente TRH según criterio de los médicos personales de las mujeres. La proporción de mujeres adherentes a la TRH se redujo del $81 \%$ en el primer año al $45 \%$ en el sexto.En el grupo placebo, las que recibieron TRH abierta fueron el $0 \%$ en el primer año y $8 \%$ en el sexto.

Medición de resultados principales

El resultado principal fue infarto de miocardio (IAM) no fatal y muerte por EC.

\section{Resultados}

No hubo reducción de eventos de EC o eventos secundarios en las mujeres que recibieron $\mathrm{TRH}$ en relación a las que recibieron placebo (ver tabla).

Tabla.Resultados principales

\begin{tabular}{|c|c|c|c|}
\hline \multirow[t]{2}{*}{ Resultado } & \multicolumn{2}{|c|}{$\begin{array}{c}\text { Nro de pacientes } \\
\text { (eventos/1000 pac/año) }\end{array}$} & \multirow[t]{2}{*}{$\begin{array}{l}\text { Riesgo Relativo } \\
\text { (IC } 95 \%)\end{array}$} \\
\hline & TRH & Placebo & \\
\hline \multicolumn{4}{|c|}{ Enfermedad Coronaria } \\
\hline HERS & $179(34.0)$ & $182(34.3)$ & $0.99(0.81-1.22)$ \\
\hline HERSII & $111(41.8)$ & $111(42.1)$ & $1.00(0.77-1.29)$ \\
\hline Global & $290(36.6)$ & $293(36.8)$ & $0.99(0.84-1.17)$ \\
\hline \multicolumn{4}{|c|}{ Muerte por EC } \\
\hline HERS & $70(12.7)$ & $59(10.6)$ & $1.20(0.85-1.69)$ \\
\hline HERSII & $62(20.6)$ & $63(20.7)$ & $0.99(0.70-1.41)$ \\
\hline Global & $132(15.5)$ & $122(14.2)$ & $1.09(0.85-1.39)$ \\
\hline \multicolumn{4}{|c|}{ IAM no fatal } \\
\hline HERS & $122(23.2)$ & $134(25.2)$ & $0.92(0.72-1.17)$ \\
\hline HERSII & $61(23.1)$ & $62(23.5)$ & $0.98(0.69-1.40)$ \\
\hline Global & $183(23.1)$ & 196 (24.7) & $0.94(0.77-1.15)$ \\
\hline
\end{tabular}

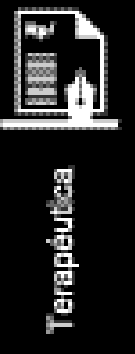

\section{Conclusiones}

Las menores tasas de EC en las mujeres que recibieron TRH en los últimos años del HERS no persistieron en el seguimiento a más largo plazo. Luego de 6.8 años de seguimiento, la TRH combinada no redujo el riesgo de eventos cardiovasculares en mujeres con EC.

Fuente de financiamiento:no referida

Un punto importante para destacar es que los eventos ocurridos en esta población fueron afortunadamente muy infrecuentes y que, aunque en términos relativos el exceso de riesgo pueda aparecer más impactante, el exceso de riesgo absoluto individual es pequeño (vide supra).

Esto implica que si aplicamos estos resultados a la práctica diaria, las mujeres que hoy están recibiendo TRH desde hace algunos años van a tener un muy pequeño aumento de riesgo de eventos adversos que rápidamente corregirá si se suspende la terapia.Este es un muy buen ejemplo de un estudio que tiene un pequeño impacto a nivel de la paciente individual pero un enorme impacto a nivel poblacional, razón por la cual provoca tal inquietud.

¿Cuál es el futuro de la TRH?4,5 En cuanto a la terapia a largo plazo, la mayoría de los autores coincide en que la indicación en la prevención primaria o secundaria cardiovascular ya no tiene ningún fundamento.Algunos discuten que la única combinación evaluada en estos estudios es la de 0,625 mg de EEC + 2,5 mg AMP en forma continua, y que otras combinaciones, dosficaciones o distintas vías de administración podrían tener resultados diferentes.Si bien es verdad que no existen estudios que evalúen el efecto del estradiol en bajas dosis por vía oral o transdérmica, el efecto de la tibolona o el agregado de otros progestágenos como la norethindrona o norgestimato, es dificil pensar en hacer un nuevo ensayo de una magnitud comparable al WHI para evaluarlos. Con respecto a la osteoporosis, no se justificaría mantener la indicación de TRH en la prevención o tratamiento cuando existen tratamientos alternativos eficaces.

En lo que respecta a la terapia a corto plazo, en las mujeres que padecen síntomas vasomotores y/o genitourinarios de intensidad moderada a severa, es sabido que no hay un tratamiento más eficaz que la TRH para lograr la mejoría.Las alternativas no hormonales no son comparables. El problema que se presenta es: ¿por cuanto tiempo deberíamos indicar TRH con este objetivo?.El mayor temor de las mujeres es sin lugar a dudas el aumento de riesgo de cáncer de mama.Los estudios muestran que parecería ser necesario un tiempo de exposición prolongado a la TRH para provocar este aumento. En este punto coinciden la gran mayoría de los estudios observacionales que han estimado el aumento relativo del riesgo en 30\% en forma consistente, con un uso superior a 3 años. En el WHI, el aumento de riesgo puede observarse a partir del tercer año de seguimiento.

En el caso de decidir iniciar TRH con este objetivo, el tiempo recomendado no debería exceder un año.Aún cuando los eventos cardiovasculares y los tromboembólicos comienzan a aumentar, el riesgo individual es extremadamente pequeño y debe ser balanceado cuidadosamente con la paciente.

Conclusión del comentario: La indicación de TRH como terapia preventiva a largo plazo tanto en prevención primaria como secundaria de eventos cardiovasculares no tiene fundamento, por lo que en aquellas pacientes que están recibiendo TRH por un largo plazo sería beneficioso suspenderla. La opción de tratamiento sintomático a corto plazo (algunos sugieren no más de un año) no parece aumentar el riesgo de cáncer de mama, ya que éste parece aumentar a partir de los 3 años de tratamiento continuo, pero se debe considerar el riesgo cardiovascular de la paciente y su decisión informada. Si bien el aumento de riesgo es considerable a nivel poblacional es muy pequeño a nivel individual, hecho que permite poder discutir la decisión con cada paciente.

Estos resultados brindan una buena oportunidad para desmedicalizar la postmenopausia y poder hablar de otras preocupaciones y miedos de las mujeres en esta etapa fisiológica de la vida. En mujeres histerectomizadas aún no esta definida la conducta ya que la rama estrógenos sin oposición vs.placebo continúa y sus resultados se esperan para el 2005.

\section{Dr. Fernando Rubinstein; Dr. Federico Augustovski [ Unidad de Medicina Familiar y Preventiva. Hospital Italiano de Buenos Aires ]}

\section{Referencias}

1. Manson J. Martin K.Post menopausal hormone replacement therapy.N Eng J Med.2001,345:34-40

2.Stevenson JC Whitehead MI. Hormone Replacement Therapy.BMJ 2002, 325:113-114

3. Hulley S, Grady D, Bush T et al. Randomized trial of estrogen plus progestin for secondary prevention of coronary heart disease in postmenopausal women. Heart and Estrogen/progestin Replacement Study (HERS) Research Group.JAMA 1998 Aug 19;280(7):605-13

4. Fletcher S, Colditz G.Failure of estrogen plus progestin therapy for prevention. JAMA 2002, 288:366-368

5. Use of combination Hormone replacement therapy in light of recent data from he Women's Health Initiative.Kaunitz A.Medscape Women's Health e journal. July 2002. 